
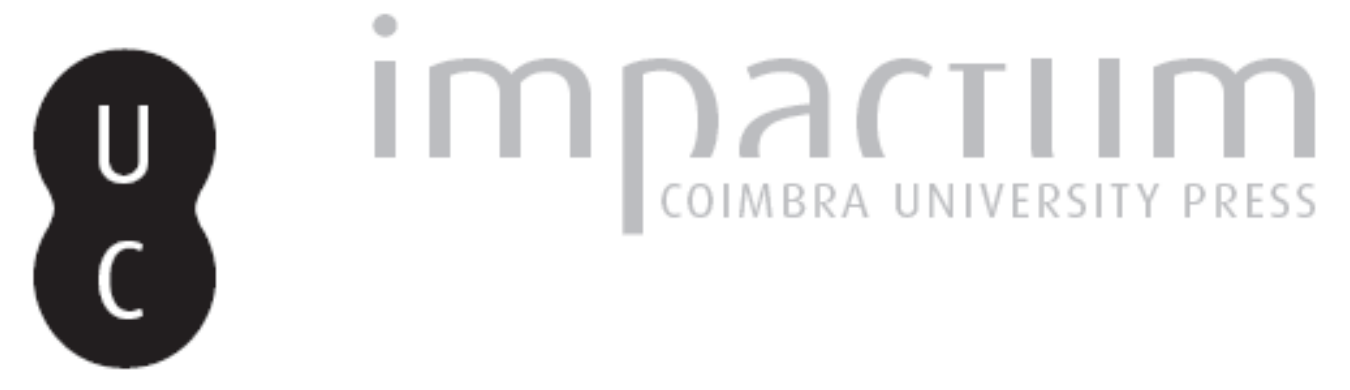

Chiralité et médicaments: une très importante découverte scientifique européenne

Autor(es): $\quad$ Lafont, Olivier

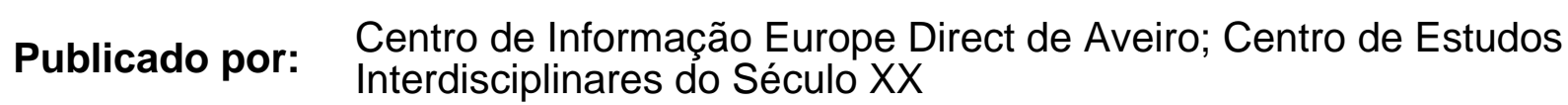

URL

persistente:

URI:http://hdl.handle.net/10316.2/39346

DOI:

DOI:http://dx.doi.org/10.14195/1647-6336_14_1

Accessed : $\quad$ 26-Apr-2023 13:42:23

A navegação consulta e descarregamento dos títulos inseridos nas Bibliotecas Digitais UC Digitalis, UC Pombalina e UC Impactum, pressupõem a aceitação plena e sem reservas dos Termos e Condições de Uso destas Bibliotecas Digitais, disponíveis em https://digitalis.uc.pt/pt-pt/termos.

Conforme exposto nos referidos Termos e Condições de Uso, o descarregamento de títulos de acesso restrito requer uma licença válida de autorização devendo o utilizador aceder ao(s) documento(s) a partir de um endereço de IP da instituição detentora da supramencionada licença.

Ao utilizador é apenas permitido o descarregamento para uso pessoal, pelo que o emprego do(s) título(s) descarregado(s) para outro fim, designadamente comercial, carece de autorização do respetivo autor ou editor da obra.

Na medida em que todas as obras da UC Digitalis se encontram protegidas pelo Código do Direito de Autor e Direitos Conexos e demais legislação aplicável, toda a cópia, parcial ou total, deste documento, nos casos em que é legalmente admitida, deverá conter ou fazer-se acompanhar por este aviso.

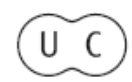


DEBATER

A EUROPA

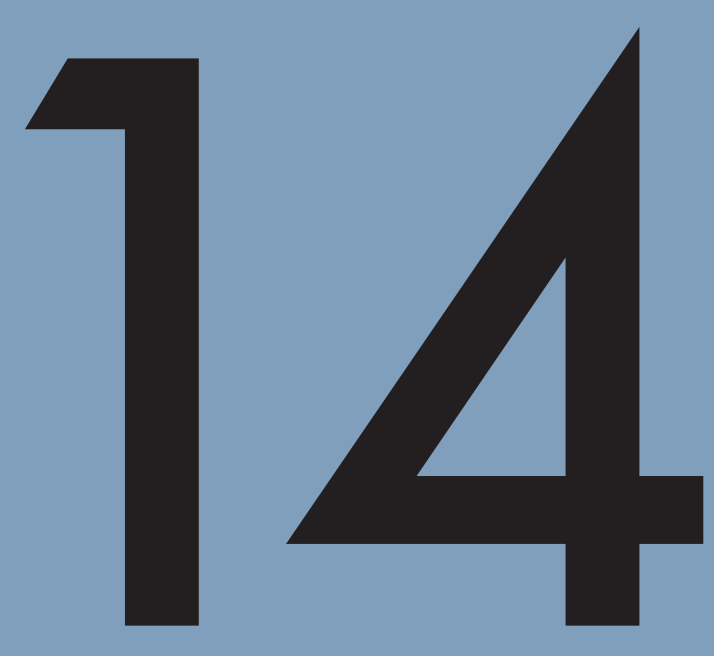

jan-jun 2016

EUROPA, FARMÁCIA, MEDICAMENTOS

EUROPE, PHARMACY, MEDICINES 


\title{
CHIRALITÉ ET MÉDICAMENTS \\ Une très importante découverte scientifique européenne
}

\author{
Olivier Lafont \\ Professor Faculté de Médecine et de Pharmacie de Rouen, France \\ Email: olivierlafont@wanadoo.fr
}

\section{Résumé}

Les travaux de Malus, de Biot, de Mitscherlich, puis de Louis Pasteur conduisirent à la découverte de la dissymétrie moléculaire, qui fut ensuite nommée chiralité par Lord Kelvin. L'importance de la chiralité pour l'activité thérapeutique des médicaments et sur leur toxicité fut progressivement mise en évidence. Les eutomères sont responsables de l'activité pharmacologique mais les distomères peuvent se révéler inactifs, ou toxiques, ou encore se montrer des auxiliaires de l'activité de l'eutomère.

Mots clefs : Chiralité ; histoire ; médicaments ; eutomères ; distomères

\section{Resumo}

Os trabalhos de Malus, de Biot, de Mitscherlich, depois de Louis Pasteur conduziram à descoberta da dissimetria molecular, que foi mais tarde denominada por quiralidade por Lord Kelvin. A importância da quiralidade para a atividade terapêutica dos medicamentos e a sua toxicidade foi progressivamente posta em evidência. Os eutómeros são responsáveis pela atividade farmacológica mas os distómeros podem revelar-se inativos, ou tóxicos, ou ainda mostrarem-se auxiliares da atividade do eutómero.

Palavras-chave: Quiralidade; história; medicamentos; eutómeros; distómeros 


\begin{abstract}
Malus, Biot, Mitscherlich and Louis Pasteur's Works conducted to the discovery molecular asymmetry, which was, later on, named chirality by Lord Kelvin. The role of chirality on pharmacological activity of medicines and on their eventual toxicity was progressively revealed. Eutomers are responsible for pharmaceutical activity, but distomers can be either devoid of any activity, toxic, or auxiliary of activity.
\end{abstract}

Keywords: Chirality; history; medicines; eutomers; distomers

Avant de pouvoir étudier la lente prise de conscience de l'importance de la chiralité dans l'activité des médicaments, il s'avère nécessaire de présenter rapidement l'historique de cette notion générale dont l'application aux molécules de la substance active des médicaments se révéla riche de conséquences.

\title{
Lumière polarisée et structure des molécules.
}

Tout débuta par une observation de physique, Étienne-Louis Malus (1775-1812) découvrit la polarisation de la lumière en 1809, en observant à travers un quartz la lumière reflétée par une lucarne ${ }^{1}$. Jean-Baptiste Biot (1774-1862) démontra alors, dès 1812, qu'un cristal de quartz découpé à angles droits pouvait dévier le plan de polarisation de la lumière préalablement polarisée. Le même Biot mit ensuite en évidence la possibilité de neutraliser l'activité optique d'une substance par celle, opposée, d'une autre. Dès 1815, il montra, sur le cas des huiles essentielles de laurier ou de citron, que des liquides pouvaient également dévier le plan de polarisation de la lumière ${ }^{2}$. Il étendit ensuite cette notion aux solutions d'un solide actif, le camphre, dans de l'alcool, un solvant totalement inactif sur la lumière polarisée ${ }^{3}$.

L'abbé René-Just Haüy (1743-1822), professeur de Minéralogie à l'École des Mines de Paris, avait, par ailleurs, dès 1801, montré que les cristaux de quartz étaient hémiédriques, c'est à dire qu'ils présentaient des facettes disposées de façon telle qu'ils n'étaient pas superposables à leur image dans un miroir ${ }^{4}$. En 1822, John Herschel (1792-1871) établit que l'activité optique des cristaux de quartz était liée à leur structure cristalline. C'était une

\footnotetext{
${ }^{1}$ MALUS, E.L - Sur une propriété de la lumière réfléchie. Mém. Soc. Arcueil. II (1809) 143-156.

2 BIOT, J.B. - Phénomènes de polarisations successives observé dans des fluides homogènes. Bull. Soc. Philpmat. (1815) 149-156.

${ }^{3}$ BIOT, J.B. - Mémoire sur la polarisation circulaire et sur ses applications à la chimie organique. Mém. Acad. Sci. 13 (1835) 39-175.

${ }^{4}$ HAÜY, [Abbé] - Traité de Minéralogie. Paris : Louis, 1801.
} 
première relation entre structure et activité optique, mais il ne s'agissait encore là que de structure cristalline ${ }^{5}$.

Biot démontra en 1835 que l'acide tartrique, isolé au siècle précédent du jus de raisin par Carl Wilhelm Scheele (1742-1786), était dextrogyre. Un isomère de cet acide tartrique, appelé acide racémique ou paratartrique, s'avérait pourtant, lui, inactif sur la lumière polarisée ${ }^{6}$. Eilhard Mitscherlich (1794-1863) remit en cause, en 1844, la liaison entre la structure et l'activité optique, car, comparant des cristaux de tartrate et de paratartrate de sodium et d'ammonium, il ne mit pas en évidence de différence dans leur structure, en dépit de leur activité totalement différente sur la lumière polarisée.

C'est alors que Pasteur intervint. L'image traditionnelle de Louis Pasteur (1822-1895) est celle d'un vieux microbiologiste penché sur ses cultures. Il fut auparavant un jeune chimiste très créatif. C'est lui qui a découvert la stéréochimie en 1848. Cela suffirait à lui assurer une place importante dans l'histoire des sciences, même si il n'avait pas, par la suite, joué le rôle que l'on sait dans le domaine de la microbiologie. Louis Pasteur observa, en effet, à l'aide d'une loupe, les cristaux d'un sel de sodium et d'ammonium de l'acide racémique et comprit que contrairement à ce que pensait Mitscherlich, il s'agissait d'un mélange de cristaux hémiédriques qu'il put séparer à l'aide d'une pince et en faire deux tas. Il vérifia alors que chaque sorte de cristaux avait une action sur la lumière polarisée, opposée à celle de l'autre. Ses conclusions furent très claires : «N'est-il pas évident maintenant que la propriété que possèdent certaines molécules de dévier le plan de polarisation de la lumière a pour cause immédiate, ou du moins est liée de la manière la plus étroite, à la dissymétrie de la molécule ? $»^{7}$.

En 1874, Joseph Achille Le Bel (1847-1930) ${ }^{8}$ et Jacobus-Henricus Van t'Hoff (18521911) ${ }^{9}$ expliquèrent, simultanément et séparément, les relations entre activité optique et structure par l'existence d'un carbone non plan, lié à quatre substituants différents. Van T'Hoff le nomma carbone asymétrique. On le représenta sous la forme d'un tétraèdre, non superposable à son image dans un miroir :

\footnotetext{
${ }^{5}$ HERSCHEL, J.W.F. - Phil.Soc.Proc. 1 (1822) 46-50.

${ }^{6}$ BIOT, J.B. - Mémoire sur la polarisation circulaire et sur ses applications à la chimie organique. Mém. Acad. Sci. 13 (1835) 39-175.

${ }^{7}$ PASTEUR, L. - Sur les relations qui peuvent exister entre la composition chimique et le sens de la polarisation de la lumière. Ann. Chim. Phys. 24 (1848) 442-459.

${ }^{8}$ LE BEL, J.A. - Sur les relations existant entre les formules atomiques des corps organiques et le pouvoir rotatoire de leurs dissolutions. Bull.Soc.Chim. II :22 (1874) 337-341.

${ }^{9}$ VAN t'HOFF, J.H. - Bull.Soc.Chim. II :23 (1875) 295-30.
} 


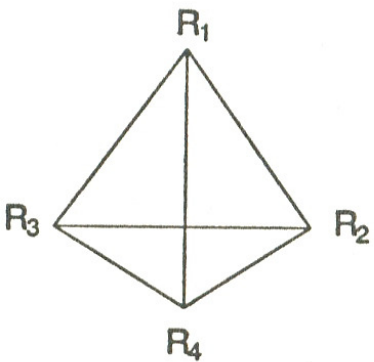

$\mathrm{R}_{4}$

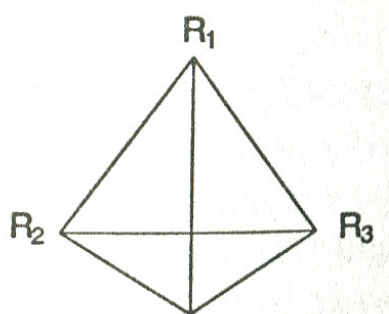

$\mathrm{R}_{4}$

On parlait alors de dissymétrie moléculaire.

À la fin du siècle, William Thomson (Lord Kelvin) (1824-1907) (10) forgea le mot « chiralité » pour désigner la propriété qui consistait à ne pas être superposable à son image dans un miroir: "I call any geometrical figure or group of points chiral and say that it has chirality if its image in a plane mirror ideally realized, cannot be brought into coincidence with itself. ». Il s'inspira du mot grec $\eta \chi \varepsilon 1 \rho$, ê cheir, désignant la main, qui est elle-même douée de cette propriété, la main droite n'étant pas superposable à la main gauche.

\section{Prise de conscience de l'importance de la chiralité des substances actives.}

Le rôle joué par la chiralité dans la vie fut reconnu très tôt. Les sucres et les acides aminés permirent en particulier de comprendre l'importance de la structure spatiale dans les processus biologiques.

On avait bien constaté que des alcaloïdes de Solanaceae, par exemple, comme l'atropine racémique et l'hyosciamine lévogyre, ne différaient que par leur stéréochimie et que cela expliquait l'activité pharmacologique supérieure de l'hyosciamine, mais on continua longtemps à mettre sur le marché, sans précaution particulière, des médicaments dont le principe actif était à l'état de mélange racémique. On se doutait bien que l'un des énantiomères, pur, aurait pu manifester une activité supérieure, mais on n'imaginait pas que l'autre énantiomère pouvait s'avérer beaucoup plus toxique.

La dramatique affaire du thalidomide fut à l'origine d'une prise de conscience de l'importance de la chiralité dans la toxicité des molécules présentes dans les médicaments.

Dans les années 1959-1962, cette molécule fut utilisée dans plusieurs pays, sous forme de mélange racémique, comme calmant léger, en particulier contre les nausées intervenant en début de grossesse. En 1962, suite à la constatation de nombreuses malformations, apparues sur des enfants de mères ayant reçu ce médicament, celui-ci fut retire du marché. 
Des études menées par la suite permirent de montrer que l'énantiomère de configuration R était seul responsable de l'activité tératogène.

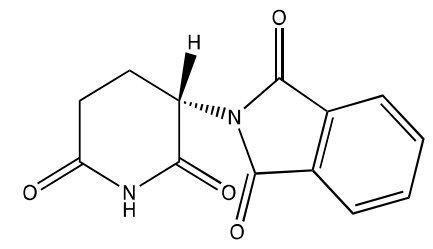

(S)-thalidomide

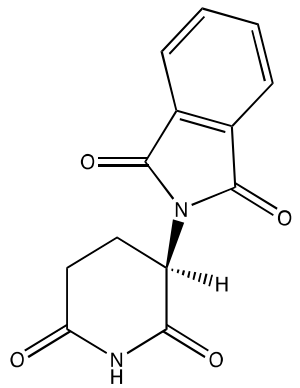

(R)-thalidomide énantiomère tératogène

On envisagea alors pouvoir utiliser l'énantiomère $S$, seul, à l'état pur. Malheureusement la présence d'un atome d'hydrogène mobile sur le centre chiral rendait possible une racémisation en milieu alcalin, ce qui ne permettait pas son utilisation en toute sécurité, en milieu biologique.

C'est à la suite de cette terrible affaire qui avait conduit à la naissance de nombreux enfants présentant une atrophie des membres supérieurs ou, dans certains cas, inférieurs également, que la législation fut modifiée et qu'il devint indispensable de faire des recherches d'activité tératogène avant toute demande d'autorisation de mise sur le marché. En cas de substance obtenue sous forme de mélange racémique, il devint également obligatoire d'effectuer des études séparées sur chaque énantiomère, à l'état pur.

\section{Eutomères et Distomères.}

Un nouveau vocabulaire vit le jour et l'on donna un nom aux énantiomères d'activité différente. Un eutomère est ainsi un énantiomère qui présente l'activité recherchée, alors qu'un distomère peut être un énantiomère inactif, moins actif, ou possédant une activité tout autre que l'activité recherchée, tout en pouvant s'avérer porteur d'une toxicité différente. Ces divers cas amènent des situations variées pour les substances concernées.

Le plus simple est celui où le distomère est simplement dépourvu de l'activité

\section{recherchée.}

À cette catégorie appartient, par exemple, le Citalopram DCI, antidépresseur, inhibiteur sélectif de la recapture de la sérotonine, d'abord commercialisé sous forme de mélange 
racémique Serplex ${ }^{\circledR}$, dont seul l'énantiomère $S$ est actif. Cet eutomère a, par la suite, été mis sur le marché sous le nom de Seroplex ${ }^{\circledR}$, ou Escitalopram ${ }_{\text {DCI. }}$

C'est le cas également du Zyrtec®, ou Cétirizine DCI, commercialisé sous forme de mélange racémique, mais dont seul l'énantiomère $\mathrm{R}$, est porteur de l'activité et celui-ci a été mis sur le marché sous le nom de Lévocétirizine ${ }_{\mathrm{DCI}}$, car il est lévogyre.

Dans le cas de la S (-) Dopa, ou Lévodopa, ou L-Dopa, un précurseur de la dopamine, c'est cet eutomère lévogyre qui est utilisé contre maladie de Parkinson.

Le distomère peut également se montrer moins actif que l'eutomère, sans être dépourvu d'activité pour autant.

Le Méthylphénidate ou Ritaline ${ }^{\circledR}$ est ainsi utilisé, sous forme racémique contre la narcolepsie chronique, les troubles de l'attention et l'hyperactivité. Cependant l'eutomère, le $d$ (thréo) Méthylphénidate ou Dexméthylphénidate ou Dextro $(\mathrm{R}, \mathrm{R})$ ritaline, plus actif que le $l$ (thréo), a également été commercialisé, sous le nom de Focaline ${ }^{\circledR}$.

Un autre groupe est constitué par les substances dont le distomère est toxique. En raison de cette toxicité, il faut impérativement ne mettre sur le marché que l'eutomère.

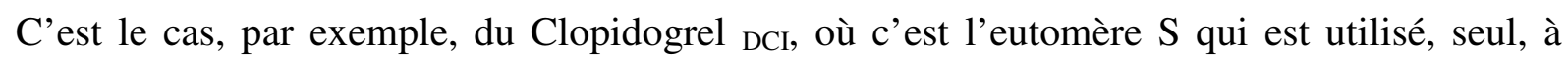
l'exclusion de son énantiomère toxique, comme antiagrégant plaquettaire actif in vivo, sous le nom de Plavix ${ }^{\circledR}$.

On peut également constater des différences de métabolisme entre deux énantiomères. L'un des énantiomères peut être lentement métabolisé, alors que l'autre l'est rapidement. C'est ainsi que, l'inhibiteur de la pompe à protons, l'Oméprazole ${ }_{\mathrm{DCI}}$ ou Mopral ${ }^{\circledR}$ est un mélange racémique. L'énantiomère $\mathrm{R}$ est rapidement métabolisé, en revanche l'énantiomère $S$, plus lentement biodégradé, possède une durée de vie plus longue et se trouve, de ce fait, responsable de l'activité. C'est pourquoi celui-ci, l'Esoméprazole ${ }_{\text {DCI, a été }}$ mis sur le marché sous le nom d'Inexium ${ }^{\circledR}$.

Des interférences entre le métabolisme ou la pharmacocinétique de deux énantiomères peuvent également se produire.

Le Méthorfane DCI en constitue un intéressant exemple. Le Dextrométhorfane constitue l'eutomère, puisqu'il est porteur de l'activité analgésique, alors que son énantiomère, le Lévométhorfane, en est dépourvu. Cependant, l'administration du racémique produit une réponse analgésique plus importante et plus durable que celle du seul Dextrométhorphane. C'est que la présence du distomère présente l'avantage de protéger l'eutomère contre la biodégradation. 
Dans le cas du Propoxyphène, c'est le R,R (+) Propoxyphène ou Dextropropoxyphène qui constitue l'énantiomère actif, alors que le Lévopropoxyphène ou S,S (-) Propoxyphène est inactif. Pourtant la présence de ce distomère augmente l'activité analgésique de l'eutomère, en provoquant sa libération de ses sites de fixation hépatique.

\section{Influence de la chiralité sur la recherche pharmaceutique.}

Lorsque l'on identifie un nouveau produit tête de série qui est chiral, plusieurs réactions sont possibles : essayer de trouver un composé non chiral équivalent, ce qui est rarement possible, effectuer des essais sur les deux énantiomères séparés pour pouvoir mettre le racémique sur le marché, mettre au point un mode de séparation rentable des énantiomères pour isoler l'eutomère en quantité importante à partir du mélange racémique, ou concevoir une synthèse stéréospécifique de l'eutomère de coût acceptable.

\section{Influence de la chiralité sur le développement pharmaceutique.}

Lorsqu'un médicament commercialisé sous forme racémique est parvenu à la fin de la durée d'exploitation de son brevet, et devient susceptible d'être présenté sous forme de générique, il est tentant pour l'entreprise qui l'a développé de mettre sur le marché l'eutomère seul, même lorsque le distomère ne présente aucun effet indésirable, en se basant sur le fait qu'il faudra toujours une quantité moindre de l'eutomère pur, pour avoir la même activité que le racémique. Il se montrera donc plus efficace pour une masse équivalente. Ce type de comportement a reçu le nom imagé de «chiral switch » ou glissement chiral.

Le chemin fut long entre la mise en évidence d'une propriété physique de la lumière, puis la découverte de son lien avec la structure des molécules, jusqu'à la mise en évidence de son importance biologique, mais la chiralité constitue désormais un facteur incontournable, pris systématiquement en compte, lors de l'étude des médicaments potentiels avant leur mise sur le marché. C'est un excellent exemple d'une notion fondamentale dont la découverte ne suscita, à l'origine, qu'un intérêt théorique et dont les applications au domaine du vivant se sont révélées par la suite d'une importance considérable. 


\section{Références}

MALUS, E.L - Sur une propriété de la lumière réfléchie. Mém. Soc. Arcueil. II (1809) 143-156.

BIOT, J.B. - Mémoire sur un nouveau genre d'oscillations que les molécules de la lumière éprouvent en traversant certains cristaux. Mém. Inst. I (1812) 372.

BIOT, J.B. - Phénomènes de polarisations successives observé dans des fluides homogènes. Bull. Soc. Philpmat. (1815) 149-156.

BIOT, J.B. - Mémoire sur la polarisation circulaire et sur ses applications à la chimie organique. Mém. Acad. Sci. 13 (1835) 39-175.

HAÜY, [Abbé] - Traité de Minéralogie. Paris : Louis, 1801.

HERSCHEL, J.W.F. - Phil.Soc.Proc. 1 (1822) 46-50.

PASTEUR, L. - Sur les relations qui peuvent exister entre la composition chimique et le sens de la polarisation de la lumière. Ann. Chim. Phys. 24 (1848) 442-459.

LE BEL, J.A. - Sur les relations existant entre les formules atomiques des corps organiques et le pouvoir rotatoire de leurs dissolutions. Bull.Soc.Chim. II :22 (1874) 337-341.

VAN t'HOFF, J.H. - Bull.Soc.Chim. II :23 (1875) 295-30.

THOMSON, W. - Baltimore Lectures, 1884 and 1893. London: Clay and Conty, 1904.

Artigo Recebido a 18 de setembro de 2015 I Aceite a 02 de março de 2016 